\title{
In Vitro and in Silico Evaluation of the Potential for Neuroprotection of Rhodiolife, A Rhodiola Rosea Roots Extract
}

\author{
José M Zubeldia (Corresponding author)
}

Development, PoliNat SL

Taibique 4, Polígono Industrial Las Majoreras, 35240 Las Palmas, Spain

Tel: 34-92-873-4132Ｅ-mail: jose@polinat.com

\author{
Aarón Hernández-Santana \\ Research, PoliNat SL
}

Taibique 4, Polígono Industrial Las Majoreras, 35240 Las Palmas, Spain

Tel: 34-92-873-4132Ｅ-mail: aaron@polinat.com

Miguel Jiménez del Rio

Management \& Production, PoliNat SL

Taibique 4, Polígono Industrial Las Majoreras, 35240 Las Palmas, Spain Tel: 34-92-873-4132_E-mail: mjimenez@polinat.com

\section{Verónica Pérez-López}

Nutragenomics laboratory, PoliNat SL

Taibique 4, Polígono Industrial Las Majoreras, 35240 Las Palmas, Spain Tel: 34-92-873-4132_E-mail: veronica@polinat.com

Received: Oct. 6, 2013 Accepted: November 28, $2013 \quad$ Published: December 1, 2013 doi:10.5296/jfs.v2i2.4632 URL: http://dx.doi.org/10.5296/jfs.v2i2.4632 


\begin{abstract}
Rhodiola rosea, an adaptogen plant from cold regions, has been previously proposed for alleviating dementia and other neurodegenerative diseases. The goal of our study was to evaluate if our proprietary extract (RhodioLife) was able to elicit biological responses related to neuroprotection in neuronal cultures. NS20Y cells were cultured according to procedures and increasing concentrations of RhodioLife were added to the media. Viability at $24 \mathrm{~h}$ using Presto Blue ${ }^{\mathrm{TM}}$ showed no statistically significant differences at those concentrations (0-50 ppm). Quantitative real-time RT-PCR analysis (G-coupled protein receptor [GCPR] array) showed statistically significant $(\mathrm{p}<0.05)$ upregulation of 3 genes: calcitonin receptor-like (CALCRL), cyclin-dependent kinase inhibitor 1A (CDKN1A), and lysophosphatidic acid receptor 2 (LPAR2) (4, 3 and 2-fold respectively). In silico evaluation of the bioactives contained in RhodioLife (www.molinspiration.com) revealed that Salidroside, Rosarin, Rosavin, Rosiridin, Cinnamyl alcohol and p-Tyrosol all had 1 or no violations of the Lipinski's rule of five, suggesting favorable pharmacokinetics. The predicted G-coupled protein receptor bioactivity was greatest for Rosarin (0.39) and Salidroside (0.35). We conclude that RhodioLife contained substances which had relevant biological activity and molecular properties suggesting a role in neuroprotection. Studies in suitable animal models are recommended.
\end{abstract}

Keywords: Neurodegenerative disease, Rhodiola rosea, G-coupled protein receptor, Quantitative real-time RT-PCR analysis 


\section{Introduction}

As the world population grows older and lifespan increases the prevalence of dementia and other neurodegenerative disorders increases. More than 24.2 million people worldwide were estimated to have dementia by 2005 and 4.6 millions of new cases have been diagnosed every year since. Although prevalence is highest in North America and Western Europe, developing countries are not free of the disease either. The overall prevalence in Africa has been reported to reach $2.4 \%$ of those older than 50 . Both westernized societies and developing countries share the common traits of increasing prevalence with increasing age (George et al, 2012; Reitz et al 2011).

It is clear that cognitive decline is a mayor public health issue and its monetary costs cannot be overlooked. In 2010 researchers calculated a cost per person between $\$ 41,689$ and $\$ 56,290$ per year which amounted to a total of $\$ 157$ to $\$ 215$ billion in the United States alone (Hurd et al, 2013). Similar overall estimated costs have been also published in Europe (Luengo-Fernández et al, 2011). Until new effective therapies are brought into the regular clinical care preventive interventions, including nutrition are of the utmost importance.

Rhodiola rosea (RR), also known as Aaron's rod, is a perennial plant typical of very cold, harsh climatic conditions located in areas at altitudes $1,800 \mathrm{~m}$ above sea level in Europe and Asia. Traditional uses include increasing resistance to stress, aiding in mental acuity or ameliorating high altitude sickness amongst many others (Kelly, 2001). Biochemical investigations have shown that an alcoholic extract of RR can cause inhibition of acetylcholine esterase (AChE) explaining its mental and memory enhancing properties. Specifically, hydroquinone, gossypetin-7-O-L-rhamnopyranoside and rhodioflavonoside have been implicated in this inhibition and have been proposed as the basis for treating Alzhemier's dementia (Hillhouse et al, 2004, Wang et al, 2007). More recently Lee et al have also reported that rosin was able to reduce the neurotoxicity induced by L-glutamine in neuronal cells. Additionally, L-glutamine increased the level of phosphorylated MAPK, pJNK and pp38, but treatment with Rosin and Salidroside resulted in a decrease indicating the potential for RR for the treatment of inflammation and neurodegeneration (Lee et al, 2013).

Our aims were to explore if our proprietary RR extract (RhodioLife) showed in vitro biological activity related to protection in dementia and neurodegeneration and investigate the molecular plausibility of acting in the central nervous system.

\section{Materials and methods}

\subsection{Rhodiola rosea extract preparation}

Approximately 5 year old Rhodiola rosea roots were obtained from the Altai region in Siberia, cleaned, cut, dried, and sieved. A water:ethanol extraction process under controlled temperature was later applied followed by filtration, distillation, concentration and drying. The chromatographic analysis of the final product showed a total Rosavins content of $>3 \%$ and salidroside $>1 \%$. 


\subsection{Cell culture}

A mouse neuroblastoma cell line, NS20Y (American Type Culture Collection, UK), was cultured in Dulbecco's Modified Eagle's Medium ( HyClone DMEM/High Glucose) supplemented with 5\% fetal bovine serum (Lonza, Belgium), 5\% calf serum, 1\% L-Glutamine $200 \mathrm{mM}$ and $1 \%$ penicillin/streptomycin (PAA, Austria). Cells were maintained at $37^{\circ} \mathrm{C}$ in a humidified $5 \% \mathrm{CO}_{2}$ incubator and medium was replaced every day.

\subsection{Cell Viability}

Cell viability was determined using the Presto Blue cell viability kit (Invitrogen, Spain) following the manufacturer's instructions. The cells were seeded into 96-well plates at a density of $6 \times 10^{4}$ cells $/ \mathrm{ml}$. The culture medium was replaced with DMEM containing various concentrations of Rhodiolife ${ }^{\circledR}$ for 1, 24, 48 and $72 \mathrm{~h}$. Control cells were cultured in DMEM without extract. Before Presto Blue kit reagents were added, the medium was removed. The cells were incubated with Presto Blue for $1 \mathrm{~h}$ at $37^{\circ} \mathrm{C}$. Fluorescence was measured on a MX3005P Q-PCR System (Agilent Technologies, Spain) using a Cy3 filter set on plate read mode.

\subsection{RNA and Protein extraction and Quantitative real-time RT-PCR analysis}

NS20Y cells were incubated with 50ppm Rhodiolife ${ }^{\circledR}$ for $24 \mathrm{~h}$. RNA and protein were extracted using All Prep RNA/Protein Kit (Qiagen, Spain). RNA was stored at $-80^{\circ} \mathrm{C}$ and protein at $-20^{\circ} \mathrm{C}$ until further use.

Total RNA was quantified using a fluorometric method with Quant-iT kit (Invitrogen, Spain). cDNA was reverse-transcribed from the RNA extract using RT ${ }^{2}$ First Stand cDNA kit and a $\mathrm{RT}^{2}$ Profiler PCR Array was used to analyze a panel of 84 genes representative of and involved in G-Protein Coupled Receptor-mediated signal transduction pathways (Qiagen, Spain). Quantitative real-time RT-PCR was carried out using a SYBR-Green/ROX detection in a MX3005P Q-PCR System. Samples were heated at $95^{\circ} \mathrm{C}$ for $10 \mathrm{~min}$, followed by a second stage composed of $15 \mathrm{sec}$ at $95^{\circ} \mathrm{C}, 1 \mathrm{~min}$ at $60^{\circ} \mathrm{C}$ which was repeated 40 times and third stage for dissociation curve composed of $1 \mathrm{~min}$ at $95^{\circ} \mathrm{C}, 30 \mathrm{sec}$ at $55^{\circ} \mathrm{C}$ and $30 \mathrm{sec}$ at $95^{\circ} \mathrm{C}$.

To analyze the PCR-array data, an MS-Excel sheet with macros was downloaded from the manufacturer's website (http://www.sabiosciences.com). This program calculated relative gene expression and statistical significance.

\subsection{In silico evaluation}

SMILES formulas of main bioactive compounds found in Rhodiola Rosea were uploaded into Molinspiration engine (www.molinspiraiton.com) from where molecular characteristics and predicted bioactive data were calculated and downloaded for tabulation.

\section{Results and discussion}

NS20Y is a cholinergic cell line derived from mouse neuroblastoma C1300. This cell line is a well-established in vitro model to study neuronal functions. The cells multiply rapidly in vitro, 
yet exhibit many properties characteristic of differentiated neurons (Nelson et al, 1969). In this context, NS20Y were used to study cell viability and changes in modulation of genes involved in GPCR-mediated signal transduction pathways upon treatment with RhodioLife. The normal biological processes regulated by GPCR include, but are not limited to, behavioral and mood regulation, autonomic nervous system transmission, inflammation and immune system regulation, vision and smell.

NS20Y were treated with Rhodiolife at concentrations ranging from 0.1 to $50 \mu \mathrm{g} / \mathrm{mL}$ for up to $72 \mathrm{~h}$, and cell viability was evaluated using PrestoBlue cell viability kit. This reagent is a resazurin based, membrane permeable solution that is reduced to resofurin by metabolically active cells. Resofurin is a fluorescent compound which can be quantitatively measured to determine viability (Lall et al, 2013). We did not observe any significant loss of cell viability for the range of concentrations and treatment periods in this study $(\mathrm{p}>0.05)$.

Gene modulation profiles showed statistically significant upregulation of 3 genes (Table 1). These genes belong to the G-coupled protein receptors (GCPR). Studies have found alterations in various GPCRs and their downstream signaling in Alzheimer's disease (Suo \& $\mathrm{Li}, 2010)$. The calcitonin receptor-like (CALCRL; fold $>4, \mathrm{p}<0.05$ ) binds in conjunction with receptor activity modifying protein type 1 RAMP1 to form calcitonin gene related peptide (CGRP) which is widely expressed in central and peripheral nervous system (Edvinsson et al, 2011). CGRP is suggested to be involved in an increasing number of biological activities including regulation of the blood vessels tone (Brain \& Cox, 2006). Interestingly it has been postulated that deposition of beta-amyloid plaques (a hallmark of Alzheimer's disease) exhibits a potent vasoconstrictor effect in rat microvasculature but cannot prevent CGRP from inducing vasodilatation (Khalil et al, 1996).

Table 1. Gene modulation pattern induced in NOY2 cells treated with RhodioLife

\begin{tabular}{|l|l|l|l|}
\hline Gene & Name & \multicolumn{1}{|c|}{ Fold } & \multicolumn{1}{c|}{ P } \\
\hline Calcrl & calcitonin receptor-like & 4,2022 & 0,02002 \\
\hline Cdkn1a & cyclin-dependent kinase inhibitor 1A (p21, Cip1) & 2,992 & 0,034467 \\
\hline Lpar2 & lysophosphatidic acid receptor 2 & 2,0722 & 0,012122 \\
\hline
\end{tabular}

The cyclin-dependent kinase inhibitor $1 \mathrm{~A}$ (CDKN1A) was also upregulated (fold $>2.9$; $\mathrm{p}<0.05)$. The encoded protein functions as a regulator of cell cycle progression at G1 by binging and inhibiting the activity of cyclin-CDK2 or -CDK4 complexes (Sherr \& Roberts, 1995). Although brain cells have been traditionally considered terminal cells (that is, not being able to enter the cell cycle), experimental data have shown that cell cycle mediators such as cyclin dependent kinases (CDKs) remain in the neurons under some form of inactivation (Tamaru et al, 1993). Further work on the role of these mediators indicates CDKs modulate stress-induced apoptosis through initiation of a cell cycle and using CDK-inhibitors led to suppression of cell death and protection of neurons in several models (Kranenburg et al, 1996). 


\section{MInstitute Macrothink $_{\text {Ins }}$}

Finally, the lysophosphatidic acid receptor 2 gene (LPAR2) was also up-regulated significantly. This protein is a lysophosphatidic acid (LPA) receptor and is tightly involved in Calcium mobilization (An et al, 1998). Calcium has different but fundamental roles in neuronal plasticity and as a second messenger has been shown to participate in regulation of gene expression, energy production, membrane excitability, synaptogenesis and synaptic transmission amongst many others (Kawamoto et al, 2012). Studies in brain cells and mice have shown the expression of presenilin and amyloid precursor protein (typical pathology features of the Alzheimer's dementia) later calcium homeostasis causing synaptic dysfunction (Woods \& Padmanabhan, 2012). Senile plaques made of $\beta$-amyloid proteins accumulated over course of de development of this neurodegenerative disease further disrupts calcium homeostasis as well which later induce membrane disruption and formation of reactive oxygen species (Kawahara et al, 2011).

The second part of our experiment was to assess the plausibility of any of the bioactives actually reaching the central nervous system (CNS). Adaptogenic activity related to Rhodiola rosea has been traditionally attributed to the presence of four principal active ingredients: salidroside, rosin, rosavin and rosarin (Sokolov et al, 1985; Furmanova et al, 1998). These compounds are found at relatively high concentrations in the roots of this plant $(>0.5 \%-3 \%$; $\mathrm{w}: \mathrm{w}$ ), and are frequently employed for the standardization of commercial products (Markus et al, 2001). Extracts used in most clinical trials are standardized to minimum 3\% total rosavins and $0.8-1 \%$ salidroside, as the naturally occurring ratio of these compounds in the plant rhizomes is approximately 3:1 (Brown et al, 2002). Other biologically active substances found in Rhodiola rosea include fl avonoids (rhodiolin, rhodionin, rhodiosin, tricin, rhodalgin and acetylrhodalgin), monoterpenes (rosiridol and rosiridin), phenylmethanoids (benzyl alcohol O- $\alpha$ - L-arabinopyranosyl- $(1 \rightarrow 6)-\beta$-D-glucopyranosyde and phenyl methyl O- $\alpha$-L-arabinofuranosyl-( $1 \rightarrow 6)-\quad \beta$-D-glucopyranosyde), phenolic acids (gallic acid, chlorogenic acid, caffeic acid and hydroxycinnamic acid) and cyanogenic glycosides (rhodiocyanoside A and lotaustralin) (Ma et al, 2011; Avula et al, 2009).

Table 2. Molecular properties and predicted bioactivity of key compounds

\begin{tabular}{|l|l|l|l|l|l|}
\hline Bioactives & LogP & TPSA & NV & GCPR & MV $\left(\AA^{\mathbf{3}}\right)$ \\
\hline Catechin & 1,369 & 110,374 & 0 & 0,41 & 244,141 \\
\hline Rosarin & $-0,216$ & 158,304 & 1 & 0,39 & 375,677 \\
\hline Salidroside & $-0,701$ & 119,608 & 0 & 0,35 & 265,804 \\
\hline Chlorogenic acid & $-0,435$ & 164,744 & 1 & 0,29 & 296,267 \\
\hline Rosin & 0,325 & 99,38 & 0 & 0,23 & 296,319 \\
\hline Mongrhoside & $-1,446$ & 167,538 & 2 & 0,15 & 390,608 \\
\hline Rosavin & $-0,955$ & 158,304 & 1 & 0,13 & 375,677 \\
\hline PAC & 3,054 & 209,754 & 3 & 0,13 & 493,201 \\
\hline Rosiridin & 0,098 & 90,322 & 0 & $-0,17$ & 315,74 \\
\hline Caffeic acid & 0,941 & 77,755 & 0 & $-0,48$ & 154,497 \\
\hline Gallic acid & 0,589 & 97,983 & 0 & $-0,77$ & 135,098 \\
\hline p-Tyrosol & 1,005 & 20,456 & 0 & $-0,8$ & 133,683 \\
\hline
\end{tabular}




\section{MInstitute ${ }^{\text {Macrothink }}$}

Log P: Octanol-water partition coefficient $\log$ P. TPSA: Topological Polar surface area. NV: Number of violations of the Lipinski's rule of 5. GCPR: G-couple protein receptor. MV: Molecular volume.

Experimental approaches to improve detection of ideal candidate compounds for drug development have gained great popularity. In his 2001 article, Lipinski described how in silico estimation of solubility and permeability could help predict desirable pharmacokinetic (PK) properties and how research substances could potentially be more bio-available (Lipinski et al, 2001). Evaluation of the bioactives of RhodioLife showed most compounds with 0 violations of the Lipinski 's rule of 5, with Chlorogenic acid, Rosarin and Rosavin having 1, Mongrhoside 2 and Polymeric anthocyanins 3. Studies with pharmacokinetic (PK) analysis on Rhodiola rosea extracts are scarce in the literature, but Salidroside and Rosavin have been isolated in the plasma of human volunteers after oral administration (Panossian et al, 2010).

However these properties do not imply diffusion across the blood brain barrier (BBB) which is more difficult to predict. Topological Polar surface area has been proposed as one key molecular factor in predicting passage through the BBB, with most CNS drugs having TPSA lower than 80-90 $\AA^{2}$. However even compounds with $120 \AA^{2}$ can still have a chance of crossing it (Ghose et al, 2012). One example is fistein, a flavonoid with proven neuroprotective effects in animal models while having a TPSA of $111.123 \AA 2$. In our case (Table 2) we observed that Cinnamyl Alcohol, p-Tyrosol, Caffeic acid, Rosiridin, Gallic acid, Rosin, Catechin and Salidroside all hade TPSA lower than $120 \AA^{2}$, while having no violations of the Lipinski's rule of 5 and thus making then potential candidates.

\section{Conclusions}

We have shown that our proprietary Rhodiola rosea extract (RhodioLife) contains bioactives that are capable of stimulating gene modulation in brain cell cultures. In our model, the statistically significant expression of CALCRL, CDKN1A and LPAR2 suggests a potential role in neuroprotection. In silico evaluation of the molecular properties of the bioactives offers additional plausibility by showing a predicted favorable pharmacokinetic profile.

\section{Acknowledgment and funding}

This work was entirely funded by PoliNat S.L.

\section{Conflict of interest statement}

José M Zubeldia, Aarón Hernández-Santana, Verónica Pérez-López, and Miguel Jiménez-del-Rio work for PoliNat SL which manufactures RhodioLife.

\section{References}

An S, Bleu T, Zheng Y, \& Goetzl EJ. (1998). Recombinant human G protein-coupled lysophosphatidic acid receptors mediate intracellular calcium mobilization. Molecular Pharmacololgy, 54, 881-888.

Avula B, Wang YH, Ali Z, Smillie TJ, Filion V, Cuerrier A,Arnason JT, \& Khan IA. (2009). RP-HPLC determination of phenylalkanoids and monoterpenoids in Rhodiola rosea and identification by LC-ESI-TOF. Biomed Chromatography, 23, 865-872. http://dx.doi.org/10.1002/bmc.1198 
Brain SD, \& Cox HM. (2006). Neuropeptides and their receptors: innovative science providing novel therapeutic targets. British Journal of Pharmacology, 147. Suppl 1:S202-211. http://dx.doi.org/10.1038/sj.bjp.0706461

Brown, RP, Gerbarg P, \& Ramazanov Z. (2002). Rhodiola rosea: a phytomedicinal overview. Herbal Gram, 56, 40-52.

Edvinsson L, Eftekhari S, Salvatore CA, \& Warfvinge K. (2011). Cerebellar distribution of calcitonin gene-related peptide. (CGRP). and its receptor components calcitonin receptor-like receptor. (CLR). and receptor activity modifying protein 1. (RAMP1). in rat. Molecular and Cellular Neuroscience, 46, 333-339. http://dx.doi.org/10.1016/j.mcn.2010.10.005

Furmanowa M, Skopinska RE, Rogala E, Hartwich M. (1998). Rhodiola rosea in vitro culture - phytochemical analysis and antioxidant action. Acta Societatis Botanicorum Poloniae 67:69-73. http://dx.doi.org/10.5586/asbp.1998.009

George-Carey R, Adeloye D, Chan KY, Paul A, Kol?i? I, Campbell H, \& Rudan I. (2012). An estimate of the prevalence of dementia in Africa: A systematic analysis. Journal of Global Health, 2,20401. http://dx.doi.org/10.7189/jogh.02.020401

Ghose AK, Herbertz T, Hudkins RL, Dorsey BD, \& Mallamo JP. (2012). Knowledge-Based, Central Nervous System. (CNS). Lead Selection and Lead Optimization for CNS Drug Discovery. ACS Chemical Neuroscinece, 3, 50-68. http://dx.doi.org/10.1021/cn200100h

Hillhouse B, Ming DS, French CJ, \& Towers GHN. (2004). Acetylcholine Esterase Inhibitors in Rhodiola rosea. Pharmaceutical Biology, 42, 68-72. http://dx.doi.org/10.1080/13880200490505636

Hurd MD, Martorell P, Delavande A, Mullen KJ, \& Langa KM. (2013). Monetary costs of dementia in the United States. New England Journal of Medicine, 368, 1326-1334. http://dx.doi.org/10.1056/NEJMsa1204629

Kawahara M, Ohtsuka I, Yokoyama S, Kato-Negishi M, \& Sadakane Y. (2011). Membrane Incorporation, Channel Formation, and Disruption of Calcium Homeostasis by Alzheimer's ?-Amyloid Protein. International Journal of Alzheimers Disease, 304583. http://dx.doi.org/10.4061/2011/304583

Kawamoto EM, Vivar C, \& Camandola S. (2012). Physiology and pathology of calcium signaling in the brain. Frontiers in Pharmacology. http://dx.doi.org/10.3389/fphar.2012.00061

Kelly GS. (2001). Rhodiola rosea: a possible plant adaptogen. Alternative Medicine Reviews, 6, 293-302.

Khalil Z, Chen H, \& Helme RD. (1996). Mechanisms underlying the vascular activity of beta-amyloid protein fragment. (beta A(4).25-35). at the level of skin microvasculature. Brain Research, 736, 206-216. http://dx.doi.org/10.1016/0006-8993(96).00685-3

Kranenburg O, van der Eb AJ, \& Zantema A. (1996). Cyclin D1 is an essential mediator of apoptotic neuronal cell death. The EMBO Journal, 15, 46-54. 
Lall N, Henley-Smith CJ, De Canha MN, Oosthuizen CB, \& Berrington D. (2013). Viability Reagent, PrestoBlue, in Comparison with Other Available Reagents, Utilized in Cytotoxicity and Antimicrobial Assays. International Journal of Microbiology, 420601. http://dx.doi.org/10.1155/2013/420601

Lee Y, Jung JC, Jang S, Kim J, Ali Z, Khan IA, \& Oh S. (2013). Anti-Inflammatory and Neuroprotective Effects of Constituents Isolated from Rhodiola rosea. Evidence Based Complementary and Alternative Medicine 2013, 514049. http://dx.doi.org/10.1155/2013/514049

Lipinski CA, Lombardo F, Dominy BW, \& Feeney PJ. (2001). Experimental and computational approaches to estimate solubility and permeability in drug discovery and development settings. Advanced Drug Delivery Reviews, 46, 3-26. http://dx.doi.org/10.1016/S0169-409X(00).00129-0

Luengo-Fernandez R, Leal J, \& Gray AM. (2011). Cost of dementia in the pre-enlargement countries of the European Union. Journal of Alzheimers Disease, 27,187-196.

Ma YC, Wang XQ, Hou FF, Ma J, Luo M, Lu S, Jin P, Terevsky N, Chen A, Xu I, Pate AV, \& Gorecki D. (2011). Rapid resolution liquid chromatography. (RRLC). analysis for quality control of Rhodiola rosea roots and commercial standardized products. Natural Products Communications, 6,645-650.

Markus G, Yurdanur Y. \& Khan IA. (2001). Analysis of the marker compounds of Rhodiola rosea L.. (golden root). by reversed phase high performance liquid chromatography. Chemical and Pharmaceutical Bulletin, 49,465-467. http://dx.doi.org/10.1248/cpb.49.465

Nelson P, Ruffner W, \& Nirenberg M. (1969). Neuronal tumor cells with excitable membranes grown in vitro. Proceedings of the National Academy of Sciences USA, 64, 1004-1010. http://dx.doi.org/10.1073/pnas.64.3.1004

Panossian A, Wikman G, \& Sarris J. (2010). Rosenroot. (Rhodiola rosea).: traditional use, chemical composition, pharmacology and clinical efficacy. Phytomedicine, 17, 481-493. http://dx.doi.org/10.1016/j.phymed.2010.02.002

Reitz C, Brayne C, \& Mayeux R. (2011). Epidemiology of Alzheimer disease. Nature Reviews Neurology, 7, 137-152. Epub 2011 Feb 8. http://dx.doi.org/10.1038/nrneurol.2011.2

Sherr CJ, \& Roberts JM. (1995). Inhibitors of mammalian G1 cyclin-dependent kinases. Genes \& Development, 9, 1149-1163. http://dx.doi.org/10.1101/gad.9.10.1149

Sokolov S, Ivashin VM, Zapesochnaya G, Kurkin VA. \& Shchavlinskii AN. (1985). Studies of neurotropic activity of new compounds isolated from Rhodiola rosea. Khimiko-Farmatsevticheskii Zhurnal, 19, 1367-1371.

Suo WZ, \& Li L. (2010). Dysfunction of G protein-coupled receptor kinases in Alzheimer's disease. Scientific World Journal, 10, 1667-1678. http://dx.doi.org/10.1100/tsw.2010.154

Tamaru T, Trigun SK, Okada M, \& Nakagawa H. (1993). Identification of cells expressing a D type G1 cyclin in matured brain: implication for its role in neuronal function. Neuroscience Letters, 153, 169-172. http://dx.doi.org/10.1016/0304-3940(93)90314-B 
Wang H, Zhou G, Gao X, Wang Y, \& Yao W. (2007). Acetylcholinesterase inhibitory-active components of Rhodiola rosea L. Food Chemistry, 105, 24-27. http://dx.doi.org/10.1016/j.foodchem.2007.03.031

Woods NK, \& Padmanabhan J. (2012). Neuronal calcium signaling and Alzheimer's disease. Advances in Experimental Medicine and Biology, 740, 1193-1217. http://dx.doi.org/10.1007/978-94-007-2888-2_54

\section{Glossary}

AChE: Acetylcholine esterase

BBB: Blood brain barrier

CALCRL: Calcitonin receptor-like

CDKN1A: Cyclin-dependent kinase inhibitor 1A

CNS: Central nervous system

GCPR: G-coupled protein receptor

JNK: c-Jun N-terminal kinase

LPA: Lysophosphatidic acid

LPAR2: Lysophosphatidic acid receptor 2

MAPK: Mitogen-activated protein kinases

MV: Molecular volume

NV: Number of violations

PK: Pharmacokinetic

RAMP1: Receptor activity modifying protein type 1

RNA: Ribonucleic acid

RR: Rhodiola rosea

RT-PCR: Real time polymerase chain reaction.

SMILES: Simplified Molecular-Input Line-Entry System

TPSA: Topological polar surface area

\section{Copyright Disclaimer}

Copyright reserved by the author(s).

This article is an open-access article distributed under the terms and conditions of the Creative Commons Attribution license (http://creativecommons.org/licenses/by/3.0/). 